\title{
Estudo da variabilidade das forças de reação do solo na aquisição do andar independente em bebês
}

\author{
Giovana Levada ${ }^{1}$ \\ Paula Hentschel Lobo da Costa ${ }^{2}$ \\ ${ }^{1}$ Programa de Pós-Graduação em Fisioterapia, Centro de Ciências Biológicas e da Saúde, UFSCar - \\ Universidade Federal de São Carlos, São Carlos, SP, Brasil \\ ${ }^{2}$ Departamento de Educação Física e Motricidade Humana, Centro de Ciências Biológicas e da Saúde, \\ UFSCar - Universidade Federal de São Carlos, São Carlos, SP, Brasil
}

\begin{abstract}
Resumo: Este estudo avaliou o comportamento das forças de reação do solo (FRS) nos primeiros meses do andar independente, a fim de identificar mudanças na capacidade infantil de propulsionar e equilibrar 0 corpo em condição dinâmica. Foram avaliadas no período de três meses dez crianças com idade de 13 meses na primeira avaliação. As crianças caminharam em uma passarela de cinco metros de comprimento com duas plataformas de força embutidas. Foram estudadas magnitudes máximas e mínimas das FRS e as respectivas variabilidades. As avaliações foram comparadas através do teste não-paramétrico ANOVA de Friedman $(p<0,05)$ e a variabilidade calculada através do coeficiente de variação $(C V)$. Não ocorreram diferenças significativas nas variáveis discretas, entretanto houve redução no CV. A experiência adquirida na marcha pelas crianças não foi suficiente para provocar mudanças nas variáveis biomecânicas estudadas. Porém, a redução gradual do coeficiente de variação para as forças verticais de reação sugere desenvolvimento em direção a um padrão de marcha mais maduro.
\end{abstract}

Palavras-chave: biomecânica; marcha; crianças; cinética.

\section{A study of ground reaction forces variability during acquisition of independent gait in toddlers}

\begin{abstract}
This study aimed to describe ground reaction forces' profiles during toddler's gait at the first months of the acquisition phase of independent walking, in order to identify whether there are changes in the ability to propel and balance the body in this dynamic condition. Ten thirteen-month old toddlers volunteered to the study and were evaluated three times in a three-month interval. The toddlers walked over a $5 \mathrm{~m}$ long walkway with two force plates fixed at ground level. Vertical and horizontal peak reaction forces and variability coefficients were selected for analysis. To compare the results of the three evaluations ANOVA Friedman's tests were used at $5 \%$ level of significance. The walking experience achieved by the toddlers month after month was not sufficient to significantly change the biomechanical selected variables and statistically significant differences were not found for the selected variables along three months. However, a reduction in the variability coefficient for the vertical reaction forces suggests development towards an increasingly mature gait pattern.
\end{abstract}

Keywords: biomechanics; gait; toddlers; kinetics.

\section{Introdução}

A aquisição da marcha pode ser considerada como o principal desafio a ser superado durante o desenvolvimento motor de um bebê (Winter, 1991). Com aproximadamente um ano de idade, bebês adotam a posição ereta e são capazes de realizar os primeiros passos independentemente (Sutherland, 1997; Grimshaw et al., 1998). O padrão do andar nessa fase está continuamente desafiado pelas características do campo gravitacional, além de ser influenciado pela carência de força muscular, por dificuldades no equilíbrio e no controle dos movimentos
(Hallemans et al., 2005). Os primeiros 4-5 meses de experiência do andar independente compõem a chamada fase de transição em que o bebê aprende a coordenar sua postura na posição vertical e o movimento dos segmentos corporais em progressão para frente (Bril \& Brenière, 1992). Nesta fase de transição, o padrão de marcha da criança é tipicamente instável, apresenta grande variabilidade (Hallemans et al., 2005; Cowgill et al., 2010) e ocorrem frequentes quedas.

Variabilidade é uma característica essencial em sistemas biológicos. Na marcha típica, a variabilidade tem sido extensamente descrita em 
variáveis biomecânicas (Hausdorff et al., 1999; White et al., 1999), bem como quando estudada em contextos de controle (Newell \& Corcos 1993) e desenvolvimento motores (Thelen, 1995). A variabilidade pode ser interpretada como uma qualidade que permite adaptação do padrão às demandas do indivíduo ou da tarefa, evidenciando que o sistema motor é capaz de selecionar uma ou outra trajetória de movimento. Comportamentos motores mais praticados são mais estáveis e evidenciam menores graus de variabilidade à medida que se tornam progressivamente mais habilidosos (Newell \& Corcos, 1993; Piek, 2002). Essa variabilidade representa uma variação funcionalmente relevante que permite o controle adaptativo do padrão de marcha.

A variabilidade pode estar relacionada à quantidade de uma dada variação (número de variações) ou à qualidade, ou seja, à capacidade para variar ou mudar. Towen (1993) afirma que durante a vida pré-natal e a infância a quantidade de variações prevalece, e a partir dessa idade segue um domínio da capacidade para variar. Os primeiros passos independentes dos bebês parecem carecer de controle, demonstrando grande variabilidade entre passos sucessivos, talvez em busca de soluções dinâmicas mais apropriadas.

O período em que a criança inicia o andar independente é caracterizado por mudanças tanto qualitativas quanto quantitativas. Ela está em processo de desenvolver e selecionar estratégias adequadas e automatizar suas escolhas (Towen, 1993). As crianças no início do andar encontramse em um período rico em mudanças que afetam diretamente o padrão do andar e a variabilidade inerente a este. O sistema motor dessas crianças precisa aprender a integrar as demandas posturais da posição ereta com as do equilíbrio dinâmico e ao mesmo tempo lidar com as mudanças em suas proporções corporais. Consequentemente, o comportamento da variabilidade durante a primeira fase do processo de aquisição do andar em crianças revela-se como um importante objeto de estudo, que pode ser analisado sob o nível cinético. As forças de reação do solo (FRS) são variáveis biomecânicas cinéticas que, como tais, aproximam a análise das causas do movimento estudado (Masani et al., 2002).
As forças de reação do solo da marcha infantil têm sido muito estudadas para fins descritivos (Takegami, 1992; Preis et al., 1997; White et al., 1999; Hallemans et al., 2006b), porém poucos estudos têm enfatizado uma abordagem longitudinal e acompanhado as mudanças que ocorrem nessas variáveis na fase inicial da aquisição do andar independente.

Dessa forma, objetivo do presente estudo foi avaliar mensalmente ao longo de três meses as forças de reação do solo e suas respectivas variabilidades durante a marcha de crianças que se encontram na primeira fase do período de aquisição, a fim de identificar se ocorrem mudanças quantitativas na capacidade de propelir o corpo adiante e um aumento da regularidade do padrão de marcha nesse período. Partindo do pressuposto de que a manifestação de padrões mais maduros de movimentos acontece quando o sistema motor responde às experiências no contexto funcional e às mudanças nas propriedades biomecânicas do organismo, espera-se verificar quantitativamente mudanças biomecânicas nos primeiros meses de aquisição do andar independente na direção de um padrão mais maduro da marcha.

\section{Métodos}

\section{Participantes}

Participaram do estudo dez crianças nascidas a termo $(39,7 \pm 0,9$ semanas $)$ e com peso ao nascer superior a $2,500 \mathrm{~g}(3,372 \pm 375 \mathrm{~g})$. Como critério de inclusão, somente foram aceitas crianças que não apresentavam desordens neuromotoras ou ortopédicas e que já andavam sozinhas há no máximo seis semanas.

Os responsáveis pelas crianças foram informados sobre a proposta e relevância do estudo e, uma vez esclarecidos, preencheram e assinaram o Termo de Consentimento Livre e Esclarecido. Foi aplicado um questionário de anamnese no início da primeira avaliação. O projeto foi aprovado e protocolado pelo Comitê de Ética em Pesquisa com Seres Humanos da universidade $\left(N^{0} 013 / 2010\right)$.

Os pais ou responsáveis por sessenta e duas crianças foram convidados a participar do estudo. Em razão de recusa ou não adequação aos critérios de inclusão apenas os responsáveis por 14 crianças compareceram na primeira avaliação. Foram eliminadas duas crianças por não comparecimento em uma das avaliações e duas 
crianças por desconforto e intolerância às coletas de dados. Dessa forma dez crianças concluíram o estudo e participaram das três avaliações.

\section{Delineamento}

Esse estudo teve caráter longitudinal. Durante o período de três meses todas as crianças foram avaliadas três vezes, com um intervalo de um mês entre as avaliações. Na primeira avaliação todas as crianças apresentavam idade cronológica de 13 meses e experiência média de andar independente de quatro semanas. $\mathrm{Na}$ segunda e na terceira avaliação as crianças apresentavam experiência média de andar independente de sete e onze semanas respectivamente. Três crianças não andaram cinco metros independentemente na primeira avaliação. Para essas crianças foi oferecido auxílio para que pudessem realizar o andar com apoio. Foram coletados dados antropométricos de cada criança em cada avaliação. A Tabela 1 apresenta a experiência (em semanas) com o andar independente de cada criança em cada avaliação.

Tabela 1. Experiência com o andar independente (em semanas) de cada criança e em cada avaliação.

\begin{tabular}{|c|c|c|c|c|c|}
\hline Sujeito & Sexo & $\begin{array}{c}\text { Idade } \\
\text { cronológica } \\
\text { na 1a } \\
\text { avaliação } \\
\text { (meses) }\end{array}$ & $\begin{array}{l}\text { Experiência no andar } \\
\text { independente na } 1^{\mathrm{a}} \\
\text { avaliação (semanas) }\end{array}$ & $\begin{array}{l}\text { Experiência no andar } \\
\text { independente na } 2^{\mathrm{a}} \\
\text { avaliação (semanas) }\end{array}$ & $\begin{array}{l}\text { Experiência no andar } \\
\text { independente na } 3^{a} \\
\text { avaliação (semanas) }\end{array}$ \\
\hline 1 & Feminino & 13 & 4 & 8 & 12 \\
\hline 2 & Masculino & 13 & 4 & 8 & 12 \\
\hline 3 & Feminino & 13 & 0 & 4 & 8 \\
\hline 4 & Masculino & 13 & 0 & 3 & 7 \\
\hline 5 & Feminino & 13 & 0 & 0 & 1 \\
\hline 6 & Masculino & 13 & 8 & 12 & 16 \\
\hline 7 & Masculino & 13 & 12 & 16 & 20 \\
\hline 8 & Masculino & 13 & 8 & 12 & 16 \\
\hline 9 & Masculino & 13 & 4 & 8 & 12 \\
\hline 10 & Feminino & 13 & 4 & 8 & 12 \\
\hline \multicolumn{2}{|l|}{ Média } & & 4,4 & 7,9 & 11,6 \\
\hline \multicolumn{2}{|c|}{ Desvio-padrão } & & $( \pm 4,0)$ & $( \pm 4,7)$ & $( \pm 5,3)$ \\
\hline
\end{tabular}

\section{Procedimentos}

Foram espalhados brinquedos no ambiente laboratorial a fim de promover descontração para a criança. Inicialmente, o avaliador realizava a familiarização com a criança e desta com o laboratório. Em seguida, as crianças andavam uma distância de cinco metros em velocidade autosselecionada. No centro da passarela estavam embutidas duas plataformas de força. A tentativa era considerada válida quando a criança pisava com um único pé em uma das plataformas e continuava sua marcha até o final da passarela. Foram consideradas para análise três tentativas válidas de cada criança. Durante as coletas, as crianças permaneceram descalças e vestidas com o mínimo possível de roupas.

\section{Análise dos dados}

Forças de reação do solo (FRS) foram coletadas por duas plataformas de força da marca Bertec (BERTEC Corporation, modelo 4060-08) dispostas em série sobre uma passarela antiderrapante de cinco metros de comprimento. Após procedimento de calibração das plataformas, os dados foram coletados a uma frequência de $100 \mathrm{~Hz}$. Os sinais das plataformas de força foram amplificados e processados por um condicionador de sinais da EMG Systems do Brasil. No programa Origin 8.1 os dados foram filtrados com filtro digital Butterworth de quarta ordem, com frequência de corte de $6 \mathrm{~Hz}$ e normalizados pelo respectivo peso corporal do participante e pela duração do apoio.

Foram selecionadas para análise as componentes vertical $(\mathrm{Fz})$ e anteroposterior (Fy) das forças de reação do solo. A componente mediolateral também foi avaliada, porém, não apresentou padrão identificável para análise devido à sua baixa magnitude e alta irregularidade. Os gráficos das FRS com as variáveis selecionadas para análise estão 
representados na Figura 1. $\mathrm{Na}$ componente vertical das FRS [Fig1(a)] o primeiro pico (contato inicial do pé com o solo) é definido como Fz1, o vale entre o valor mínimo que ocorre entre 0 primeiro e o segundo pico da componente vertical (fase de apoio médio) como Fz2 e o segundo pico (fase de pré-balanço) como Fz3. Similarmente para a componente anteroposterior, Fy1 como força anterior máxima quando o calcanhar toca o solo (frenação) e Fy2 como força posterior máxima na fase de propulsão [Fig1 (b)]. Os tempos para se atingir cada pico das variáveis da força foram definidos como tz1, tz2, tz3 (direção vertical) e ty1, ty2 (direção anteroposterior). Foi avaliada também a duração (em segundos) da fase de apoio.

(a)

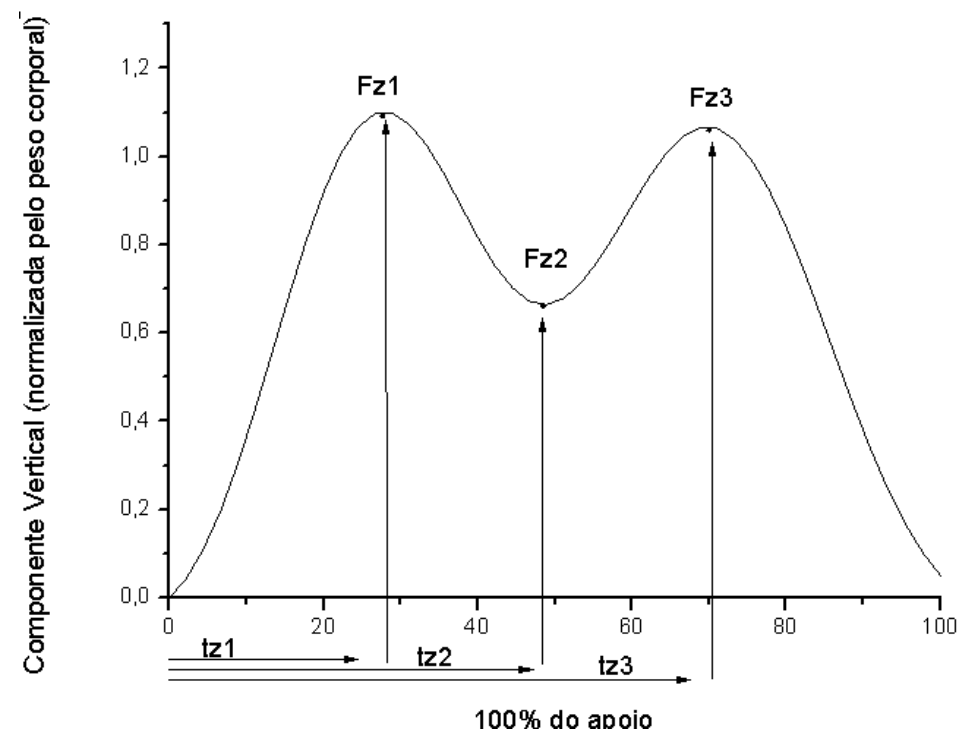

(b)

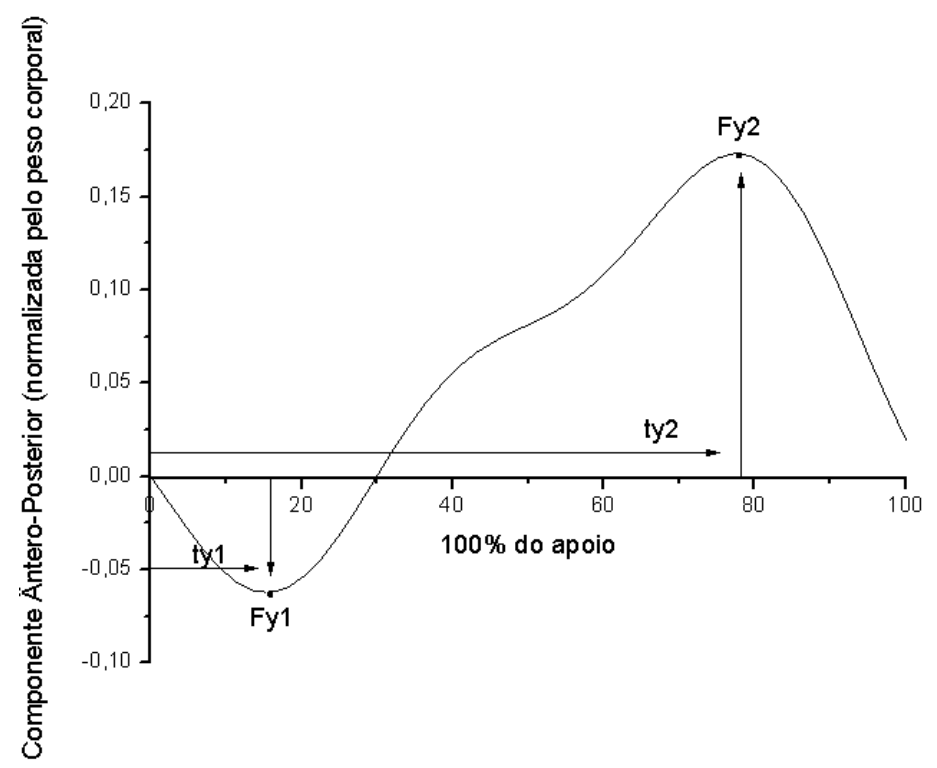

Figura 1. Gráficos selecionados das FRS das componentes vertical (a) e anteroposterior (b) de um participante.

Para o estudo do comportamento da variabilidade das forças de reação do solo, o coeficiente de variação $(\mathrm{CV})$ foi calculado entre as tentativas individuais para cada avaliação de acordo com Winter (1991):

$$
C V=\sqrt{\frac{\text { desvio padrão }}{\text { média }}} X 100
$$

O cálculo do CV a partir de Winter (1991) é interessante, pois a variabilidade é avaliada ao longo de toda a série temporal em cada tentativa 
individual da força de reação do solo e não apenas em pontos discretos selecionados.

Uma análise do coeficiente de variação entre as tentativas individuais pode indicar diferenças que caracterizam mais globalmente as curvas dinâmicas entre os diferentes indivíduos. E, ainda, por se tratar de grandezas biomecânicas de indivíduos sem disfunções motoras ou ortopédicas, as diferenças entre os coeficientes de variação provavelmente refletem a variabilidade típica do comportamento motor humano, tomados os cuidados metodológicos para o controle dos erros.

\section{Análise estatística}

Os dados foram processados pelo programa Origin 8.1. O tratamento estatístico foi realizado no STATISTICAL PACKAGE FOR THE SOCIAL SCIENCE (SPSS), versão 17.0. Para a avaliação de diferenças entre as três avaliações das variáveis discretas das forças de reação do solo e para o coeficiente de variação foi realizada uma análise de medidas repetidas não-paramétrica ANOVA de Friedman. Em seguida, para as variáveis que apresentaram diferenças, foi aplicado o post hoc de Wilcoxon. O nível de significância foi fixado em $5 \%$.

\section{Resultados}

\section{Variáveis discretas}

A Tabela 2 apresenta os valores médios e os desvios-padrão das variáveis selecionadas das forças de reação do solo em suas componentes vertical e anteroposterior para cada avaliação.

A Tabela 3 apresenta os valores médios e os desvios-padrão dos parâmetros temporais das FRS em porcentagem do tempo total de apoio e também os valores médios e os desvios-padrão para a duração da fase de apoio em cada avaliação.

Tabela 2. Valores médios (desvios-padrão) das variáveis discretas das forças de reação do solo, normalizadas pelo peso corporal.

\begin{tabular}{cccc}
\hline Variáveis & $\begin{array}{c}\mathbf{1}^{\mathbf{a}} \text { avaliação } \\
\text { (\%PC) }\end{array}$ & $\begin{array}{c}\mathbf{2}^{\mathbf{a}} \text { avaliação } \\
(\% \mathrm{PC})\end{array}$ & $\begin{array}{c}\text { 3 }^{\text {a }} \text { avaliação } \\
\text { (\%PC) }\end{array}$ \\
\hline Fz1 & $116(20)$ & $127(10)$ & $108(10)$ \\
Fz2 & $64(12)$ & $60(8)$ & $64(12)$ \\
Fz3 & $89(10)$ & $89(10)$ & $84(10)$ \\
Fy1 & $-17(7)$ & $-16(7)$ & $-13(5)$ \\
Fy2 & $18(7)$ & $17(7)$ & $15(5)$ \\
\hline
\end{tabular}

Tabela 3. Valores médios (desvios-padrão) dos parâmetros temporais, normalizados pela porcentagem da fase de apoio.

\begin{tabular}{cccc}
\hline $\begin{array}{c}\text { Parâmetros } \\
\text { temporais }\end{array}$ & 1ª avaliação & 2a avaliação & 3ª avaliação \\
\hline Tz1 (\% apoio) & $24,6(5,0)$ & $27,4(3,0)$ & $28,3(4,0)$ \\
Tz2 (\% apoio) & $47,5(5,0)$ & $50,9(3,0)$ & $49,4(7,0)$ \\
Tz3 (\% apoio) & $65,3(4,0)$ & $69,6(4,0)$ & $66,8(4,0)$ \\
Ty1 (\% apoio) & $17,9(2,0)$ & $17,9(1,0)$ & $18,1(1,0)$ \\
Ty2 (\% apoio) & $72,6(6,0)$ & $74,0(3,0)$ & $74,2(3,0)$ \\
Duração da fase de & $0,52(0,05)$ & $0,47(0,04)$ & $0,51(0,05)$ \\
apoio (s) & & & \\
\hline
\end{tabular}


Não foram encontradas diferenças significativas nas variáveis discretas e nos parâmetros temporais, entretanto é possível levantar pontos importantes. Os valores para o primeiro pico (Fz1) da componente vertical estiveram acima do peso corporal (PC), enquanto que os valores para o segundo pico (Fz3) predominaram abaixo do peso corporal (Tabela 2).

Para a componente anteroposterior, os valores estiveram entre $13 \%$ e $17 \%$ do PC para a frenação e entre $15 \%$ e $18 \%$ para o pico de propulsão (Tabela 2).

O tempo até o primeiro pico da componente vertical (Tz1) apresentou variações com valores entre $24,6 \%$ e $28,3 \%$ da fase de apoio (Tabela 3 ). A duração da fase de apoio (em segundos) não apresentou diferenças entre as avaliações. Os valores médios encontrados foram 0,52, 0,47 e 0,51 segundos para a primeira, segunda e terceira avaliação, respectivamente.

Os resultados para a componente mediolateral não apresentaram padrão identificável. Baseados nas conclusões conhecidas de Sutherland (1997), que demonstraram que a componente mediolateral da força de reação do solo, no andar de adultos, é altamente variável e que seu uso como parâmetro representativo para comparações é inapropriado, optamos por não incluir esta componente no estudo.

\section{Comportamento da variabilidade}

A Tabela 4 apresenta os valores médios e os desvios-padrão dos coeficientes de variação em cada avaliação para as componentes vertical e anteroposterior.

Tabela 4. Valores médios (desvios-padrão) dos coeficientes de variação (CV) para as curvas das componentes das FRS nas três avaliações.

\begin{tabular}{cccc}
\hline $\begin{array}{c}\text { CV das } \\
\text { componentes }\end{array}$ & $\mathbf{1}^{\circ}$ Avaliação & $\mathbf{2}^{\circ}$ Avaliação & $\mathbf{3}^{\circ}$ Avaliação \\
\hline Vertical & $71 \%(0,12)$ & $60 \%(0,12)$ & $53 \%(0,21)$ \\
\hline Anteroposterior & $248 \%(0,86)$ & $220 \%(0,42)$ & $230 \%(0,41)$ \\
\hline
\end{tabular}

O coeficiente de variação da componente vertical foi significativamente diferente entre as avaliações 1 e $3(p=0,02)$. Para a componente vertical foram encontrados valores entre $53 \%$ e $71 \%$ (Tabela 4). Houve redução do coeficiente de variação da primeira para segunda avaliação e da segunda para a terceira avaliação.

Não foram encontradas diferenças significativas nas avaliações do CV para a componente anteroposterior das forças de reação do solo. Os valores ficaram entre $220 \%$ e $248 \%$ (Tabela 4). Esses valores podem ser considerados altos quando comparados com os coeficientes de variação em estudos com crianças acima de 5 anos de idade e adultos (Winter, 1991; Lobo da Costa, 2000; David, 2000).

\section{Discussão}

Esperava-se identificar mudanças quantitativas expressas nas variáveis biomecânicas selecionadas ao longo dos três meses avaliados, em função deste período corresponder à fase inicial de aquisição, ou fase de transição, na qual ocorrem mudanças qualitativas importantes no padrão do andar infantil. Por outro lado, poucos estudos focaram esse mesmo período da aquisição da marcha e não foram encontradas avaliações de caráter longitudinal para as variáveis selecionadas para efeito de comparações adequadas. Dessa forma, a discussão dos resultados obtidos será feita com base em estudos da marcha infantil, independentemente da faixa etária.

Stansfield et al. (2001) avaliaram crianças saudáveis de cinco anos de idade a fim de estudar a importância da idade e velocidade na caracterização das forças de reação do solo. A análise da componente vertical em velocidade autosselecionada pela criança apontou valores médios para o primeiro pico (contato inicial do pé com o solo) da componente vertical entre $110 \%$ e $130 \%$ do peso corporal, valores entre $60 \%$ e $85 \%$ para o vale (fase de apoio médio) e valores entre $100 \%$ e $120 \%$ para o segundo pico (fase de prébalanço). Semelhanças com o nosso estudo são encontradas nos valores para o primeiro pico e vale da componente vertical, entretanto no nosso estudo os valores para o segundo pico ficaram 
abaixo do peso corporal (Tabela 2), sugerindo baixa capacidade de propelir o corpo à frente destas crianças. Sutherland (1997) também identificou valores abaixo do peso corporal para a fase de pré-balanço da componente vertical em crianças nas idades de dois anos a sete anos.

Com base em nossos resultados e considerando os voluntários de nosso estudo, podemos ponderar que crianças na aquisição do andar independente não possuem uma fase de propulsão efetiva e que o período de três meses usado no presente estudo para acompanhar tais mudanças não foi suficiente para evidenciá-las. É possível que o rolamento do pé no solo durante a fase de apoio ainda não esteja completo nessa fase (Hallemans et al., 2006a), o que pode limitar a propulsão contra o solo.

A duração da fase de apoio foi usada no presente estudo como preditora da velocidade de deslocamento, pois sabe-se que estas são variáveis altamente relacionadas quando se tem cadência regular (Andriacchi et al., 1977). Os tempos de apoio encontrados são semelhantes aos resultados de Grimshaw et al. (1998) que estudaram o andar de crianças de 10 a 24 meses de idade, além disso, não foram encontradas diferenças significativas nesta variável ao longo dos três meses de estudo.

A média dos valores para a duração da fase de apoio entre as avaliações foi de 0,50 segundos com um desvio-padrão de 0,02, o que corresponde a $5,2 \%$ de variação entre as avaliações. Assim, apesar de acreditarmos que nossas crianças não andaram em cadência regular e admitirmos a grande dificuldade desse controle, entendemos que não houve diferenças nas velocidades de deslocamento entre as tentativas usadas para a análise dos dados e, assim, velocidades variáveis não influenciaram os resultados da cinética e dos coeficientes de variabilidade deste estudo.

As magnitudes da componente anteroposterior no padrão maduro da marcha variam em torno de $20 \%$ do peso corporal tanto na frenagem quanto na propulsão (BECK et al., 1981; Takegami, 1992). Apesar de alguns dos nossos resultados alcançarem magnitudes em torno de $20 \%$ do peso corporal, a média para o pico de frenação esteve entre 13 e $17 \%$ e para o pico de propulsão entre 15 e $18 \%$, o que sugere que as crianças de nosso estudo ainda não apresentem uma forma efetiva de realizar a frenação e a propulsão do andar.
Takegami (1992) verificou em seus resultados uma diminuição no tempo até o primeiro pico da componente vertical (Tz1). Os valores para suas crianças com quatro anos de idade foram de $25 \%$ do tempo total de apoio, enquanto que as crianças com dez anos de idade apresentaram valores para $\mathrm{Tz} 1$ de $21 \%$ do tempo total de apoio. Essa redução em Tz1 é considerada pelo mesmo autor como uma melhora na capacidade para transferência de cargas nos pés que ocorre à medida que as crianças adquirem maior experiência com o andar. Nossos valores ficaram entre $24,6 \%$ e $28,3 \%$, apontando variações no tempo até o primeiro pico (Tabela 3). Esse resultado nos permite levantar a possibilidade de que nossas crianças, em período de aquisição do andar independente, encontram-se em pleno aprimoramento da transferência de cargas no apoio durante a fase de rolamento do pé, e devem executar o rolamento do pé sobre solo de diferentes maneiras (Hallemans et al., 2006a), sem uma preferência definida para a execução do apoio.

O coeficiente de variação (CV) foi usado para determinar a variabilidade das forças de reação do solo. O CV tem a vantagem de ser uma ferramenta simples de obter reprodutibilidade desse tipo de dados (White et al., 1999).

Foram encontrados poucos estudos que utilizaram o mesmo cálculo do CV. Os trabalhos encontrados foram realizados com crianças acima de cinco anos de idade e adultos (Winter, 1991; Lobo da Costa, 2000; David, 2000).

Winter (1991) demonstrou que o CV para indivíduos adultos das forças verticais em cadência natural é de $18 \%$, enquanto que para as forças horizontais é de 43\%. David (2000) avaliou crianças com idades entre 6 e 10 anos e encontrou valores para a componente vertical entre $9 \%$ e $17 \%$ e valores entre $28 \%$ e $37 \%$ para a anteroposterior. Lobo da Costa (2000) encontrou valores entre $21,1 \%$ e $51,1 \%$ para forças verticais e entre $21,6 \%$ e $115 \%$ para a componente anteroposterior em crianças com idade média de 6,5 anos. Estes valores estão abaixo dos nossos resultados, apesar de manterem a tendência de menores variabilidades para a componente vertical, comparada à horizontal anteroposterior.

O nosso estudo encontrou valores entre $53 \%$ e $71 \%$ para o CV da componente vertical e valores entre $230 \%$ e $248 \%$ (Tabela 4) para o CV da componente anteroposterior. Esses valores 
podem ser considerados altos quando comparados aos estudos citados anteriormente, fato que pode ser atribuído às diferentes idades dos indivíduos nos respectivos estudos.

Podemos afirmar que o padrão do andar no período de aquisição é altamente variável. Estatisticamente não foram encontradas diferenças significativas para $\mathrm{C} C \mathrm{CV}$ da componente anteroposterior. Entretanto, para a componente vertical houve diferença estatística entre a primeira e a terceira avaliação. Esse resultado é evidência de que o intervalo de um mês entre as avaliações não foi suficiente para provocar mudanças significativas na variabilidade na fase de aquisição do andar independente, porém, três meses, entre a primeira e a última avaliação, foram suficientes e provocaram mudanças na variabilidade da componente vertical das forças de reação do solo, levando o padrão do andar a um comportamento gradualmente mais regular.

Para a componente anteroposterior os valores para o $\mathrm{CV}$ podem ser considerados altos quando comparados com os valores encontrados na literatura (Winter, 1991; Lobo da Costa, 2000; David, 2000). Nós não acreditamos que esses valores sejam erros de medida, mas sim uma característica da aquisição da marcha independente. Entretanto, não encontramos estudos que utilizaram o cálculo do CV a partir de Winter (1991) para análise da marcha em crianças no início do andar independente. Por isso, estudos posteriores e longitudinais com crianças típicas devem ser realizados a fim de caracterizar melhor a variabilidade esperada na fase de aquisição do andar e obter um critério fisiológico para que se possa, por exemplo, avaliar futuramente crianças com desordens motoras.

A partir dos valores apresentados para o CV das forças de reação do solo, concluímos que a variabilidade encontrada é realmente devida ao nível funcional do comportamento motor infantil na fase de aquisição do andar independente, nível este que parece começar a se alterar apenas para a componente vertical nos primeiros meses do andar independente, sendo que a componente anteroposterior não teve sua variabilidade alterada no mesmo período. Assim, parece-nos que a componente vertical, por melhor refletir a demanda antigravitacional imposta à criança ao ter que sustentar o corpo apropriadamente para ser capaz de propulsioná-lo à frente, é a primeira a responder em termos desenvolvimentistas.

Entre o décimo segundo e o décimo quinto mês de vida a maioria das crianças consegue andar completamente sem auxílio (Sutherland, 1997; Grimshaw et al., 1998). Após esse marco, o processo de refinamento do padrão do andar continua, com um ritmo de desenvolvimento não estável na aquisição das habilidades motoras, podendo haver períodos em que nenhuma habilidade motora seja adquirida e outros com grandes saltos qualitativos (Thelen \& Ulrich, 1991). Todas as crianças do presente estudo encontravam-se nessa fase, porém destaca-se que uma observação qualitativa do comportamento das nossas crianças no ambiente laboratorial revelou que estas possuíam experiências diferentes com 0 andar independente mesmo tendo a mesma idade cronológica durante as avaliações.

Os diferentes graus de experiência com a marcha em cada criança, conhecidos através do relato dos pais, devem ter influenciado nossos resultados e dificultado a identificação de diferenças significativas entre as variáveis selecionadas ao longo dos três meses avaliados.

A estimulação doméstica e a soma das oportunidades para a exploração do ambiente e de seus recursos motores não estão necessariamente refletidas na idade cronológica, o que torna esta um fraco preditor do "status" de desenvolvimento do andar infantil. Talvez mais adequado seja classificar um grupo de crianças com base no grau de experiência com o andar, o que poderia ser feito através de uma escala de desenvolvimento e, a partir dessa distribuição de grupos, acompanhar mudanças nas variáveis biomecânicas.

Outra limitação do nosso estudo diz respeito à dificuldade de se obter total controle das condições experimentais, em função da faixa etária das crianças. A literatura (Beck et al.,1981; Hallemans et al., 2006b) reporta que para se estudar o desenvolvimento do andar infantil é necessário o controle da velocidade, a fim de se assegurar que as mudanças observadas sejam devidas ao nível de desenvolvimento e não as diferenças nas velocidades alcançadas. Entretanto, quando estamos no ambiente laboratorial nem sempre é possível uma boa colaboração da criança e, nesses casos, tanto o controle rigoroso da velocidade, quanto a 
realização bem sucedida da tentativa nem sempre ocorrem concomitantemente.

Por fim, a experiência adquirida na marcha pelas crianças ao longo do intervalo de três meses não foi suficiente para provocar mudanças estatisticamente relevantes nas variáveis biomecânicas estudadas, picos de forças de reação e seus respectivos tempos, porém houve uma redução gradual dos coeficientes de variação da componente vertical das forças de reação do solo.

Acreditamos que nossos resultados são evidências de que o aumento da capacidade de encontrar soluções dinâmicas progressivamente mais regulares e maduras deva se iniciar com o controle das demandas gravitacionais associadas à marcha. Por outro lado, o uso do critério da idade cronológica nos pareceu inadequado para se classificar o grau de desenvolvimento do andar durante a fase de aquisição da marcha em bebês, uma vez que as experiências individuais com a exploração do ambiente são cruciais nessa fase e devem melhor regular diferenças biomecânicas que ocorrem em curtos intervalos de avaliação.

\section{Referências}

ANDRIACCHI, T, P.; OGLE, J.A.; GALANTE J.O. Walking speed as a basis for normal and abnormal gait measurements. Journal of Biomechanics, New York, v.10, p.261-268, 1977.

BECK, R. J.; ANDRIACCHI, T. P.; KUO, K. N.; FERMIER, R. W.; GALANTE, J. O. Changes in the gait patterns of growing children. The Journal of Bone and Joint Surgery, Needham, v. 63-A, n. 9, p.1452-6, 1981.

BRIL, B.; BRENIERE, Y. Postural requirements and progressive velocity in young walkers.

Journal of Motor Behavior, Washington, v.24, p.105-116, 1992.

COWGILL, L.W.; WARRENER, A.; PONTZER, $\mathrm{H}$.; OCOBOCK, C. Waddling and Toddling: The biomechanical effects of immature gait. American Journal of Physical Anthropology, Philadelphia, v.143, p.52-61, 2010.

DAVID, A.C. Aspectos biomecânicos do andar em crianças: cinematica e cinética. 2000. $140 \mathrm{f}$. Tese (Doutorado em Educação Física). Universidade Federal de Santa Maria, Santa Maria, 2000.

GRIMSHAW, P.N.; MARQUES, P.; SALO, AKI.; MESSENGER, NEIL. The 3-dimensionak kinematics of the waking gait cycle of children aged between 10 and 24 months: cross sectional and repeated measures. Gait \& Posture, Oxford, v.7, p7-15, 1998.

HALLEMANS, A.; DeCLERCQ, D.; OTTEN, B.; AERTS, P. 3D dynamics of walking in toddlers: A cross-sectional study spanning the first rapid development phase of walking. Gait \& Posture, Oxford, v. 22, p. 107-118, 2005.

HALLEMANS, A.; DeCLERCQ, D.; DONGEN V.S.; AERTS, P. Changes in foot-function parameters during the first 5 months after the onset of independent walking: a longitudinal follow-up study. Gait \& Posture, Oxford, v. 23, p. 142-148, 2006a.

HALLEMANS, A.; DeCLERCQ, D.; AERTS, P. Changes in $3 D$ joint dynamics during the first 5 months after the onset of independent walking: a longitudinal follow-up study. Gait \& Posture, Oxford, v.24, p.270-279, 2006b.

HAUSDORFF, J.M.; ZEMANY, L. PENG, C.K.; GOLDBERGER, A.L. Maturation of gait dynamics: stride-to-stride variability and its temporal organization in children. Journal of Applied Physiology, Bethesda, v.86, p.1040-1047, 1999.

LOBO DA COSTA. P.H. Aspectos biomecânicos da locomoção infantil:grandezas cinéticas no andar e correr. 2000 Tese (Doutorado em Educação Física) . Faculdade de Educação Física da Universidade de São Paulo, São Paulo, 2000.

MASANI, K.; KOUZAKI, M.; FUKUNAGA, T. Variability of ground reaction forces during treadmill walking. Journal of Applied Physiology, Bethesda, v.92, p.1885-1890, 2002.

NEWELL, K. M.; CORCOS, D. M. (Eds.). Variability and Motor Control. Champaign, Human Kinetics, 1993.

PIEK, J.P. The role of variability in early motor development. Infant Behavior \& Development, Norwood, v.25, p452-465, 2002.

PREIS, S.; KLEMMS, A.; MÜLLER, K. Gait analysis by measuring ground reaction forces in children: changes to an adaptive gait pattern between the ages of one and five years.

Developmental Medicine and Child Neurology, London, v.39, p.228-233, 1997.

STANSFIELD, B.W.; HILLMAN, S.J.; HAZLEWOOD, M.E.; LAWSON, A.A.; MANN, A.M.; LOUDON, I.R.; ROBB, J.E. Normalized speed, not age, characterizes ground reaction force patterns in 5 to 12 year old children walking at self-selected speeds. Journal of Pediatric Orthopedics, New York, v.21, p.395-402, 2000. 
SUTHERLAND, D.H. The development of mature gait. Gait \& Posture, Oxford, vol.6, p.13-170, 1997.

TAKEGAMI, M.D.Y. Wave Pattern of Ground Reaction Force of Growing Children. Journal of Pediatric Orthopedics, New York,12, p.522-526, 1992.

THELEN, E. Motor Development: a new synthesis. The American psychologist, Washington, 50 (2), p.79-95, 1995.

THELEN, E., ULRICH, B.D. Hidden skills: A dynamic systems analysis of treadmill stepping during the first year. Monographs of the Society for Research in Child Development, Chicago, v.56, p.I06, 1991.

TOWEN, L.C.B. How normal is variable, or how variable is normal? Early Human Development, Amsterdam, v.34, p.1-12, 1993.

WHITE, R.; AGOURIS, I.; SELBIE, R.D.; KIRKPATRICK, M. The variability of force platform data in normal and cerebral palsy gait. Clinical Biomechanics, Oxford, v.14, p185-192, 1999.

WINTER, D. A. The biomechanics and motor control of human gait: normal, elderly and pathological. Waterloo, University of Waterloo, 1991.

\section{Endereço:}

Giovana Levada

Avenida Araújo de Brito, 32 Novo Horizonte

Nanuque MG Brasil

39860-000

Telefones: (33) 3621-4547 / (73) 8111-4865

e-mail: giulevada@yahoo.com.br 